\title{
Spin polarization of $\mathrm{Fe}_{4} \mathrm{~N}$ thin films determined by point-contact Andreev reflection
}

\author{
A. Narahara, ${ }^{1}$ K. Ito, ${ }^{1}$ T. Suemasu, ${ }^{1, a)}$ Y. K. Takahashi, ${ }^{2}$ A. Ranajikanth, ${ }^{2}$ and K. Hono $^{2}$ \\ ${ }^{1}$ Institute of Applied Physics, University of Tsukuba, 1-1-1 Tennohdai, Tsukuba, Ibaraki 305-8573, \\ Japan \\ ${ }^{2}$ National Institute for Materials Science, Tsukuba 305-0047, Japan and University of Tsukuba, Tsukuba 305- \\ 0047, Japan
}

(Received 14 April 2009; accepted 30 April 2009; published online 18 May 2009)

\begin{abstract}
The spin polarization of (100)-oriented $\gamma^{\prime}-\mathrm{Fe}_{4} \mathrm{~N}$ layers grown on $\mathrm{MgO}(001)$ substrates by molecular beam epitaxy was deduced from point contact Andreev reflection measurements, and the value was compared with that of $\alpha$-Fe. The spin polarization $(P)$ for $\gamma^{\prime}-\mathrm{Fe}_{4} \mathrm{~N}$ is approximately 0.59 at $7.8 \mathrm{~K}$. This value is distinctly larger than that for $\alpha$ - Fe $(P=0.49$ at $7.8 \mathrm{~K})$ measured with an identical setting. The mechanism of enhanced spin polarization in $\gamma^{\prime}-\mathrm{Fe}_{4} \mathrm{~N}$ is discussed. (C) 2009 American Institute of Physics. [DOI: 10.1063/1.3140459]
\end{abstract}

Highly spin-polarized ferromagnetic materials are of great interest as sources of spin currents in various spintronics devices. Numerous types of half-metals and their heterojunctions have been studied extensively since de Groot et al. ${ }^{1}$ proposed the theoretical concept of half-metals in 1983. For many practical applications in spintronics devices, the halfmetals must be ferromagnetic at room temperature. Because of this requirement, Co-based Heusler alloys, which were theoretically predicted to be ferromagnetic half-metals, have been intensively studied. ${ }^{2-8}$ However, half-metallicity is based on the total density of states (DOSs), and half-metals based on DOS do not necessarily yield highly spin polarized transport in tunneling magnetoresistance (TMR) and currentperpendicular-to-plane giant magnetoresistance (CPP GMR). Therefore, ferromagnetic electrodes that give high spin polarization in electron transport need to be sought. Recent theoretical analysis by Kokado et al. ${ }^{9}$ predicted highly enhanced spin polarization of transport electrons in ferromagnetic fcc-Fe, which motivated us to investigate iron nitrides as a potential spintronics material. Although Kokado et al. ${ }^{9}$ predicted highly spin-polarized transport in $\gamma^{\prime}-\mathrm{Fe}_{4} \mathrm{~N}$, there have been few experimental investigations. Sunaga et al. ${ }^{10}$ and Komasaki et al. ${ }^{11}$ fabricated $\mathrm{Fe}_{4} \mathrm{~N} / \mathrm{MgO} / \mathrm{CoFeB}$ magnetic tunnel junctions (MTJs), and reported negative but relatively low spin polarization of $\mathrm{Fe}_{4} \mathrm{~N}$ deduced from TMR ratios using Julliere's formula. ${ }^{12}$ It is well known that the spin polarization values deduced from this formula are of tunneling electrons and largely influenced by the interfacial structure. Hence, they are different from the intrinsic spin polarization of electrode materials. ${ }^{13}$ In contrast, point-contact Andreev reflection (PCAR) directly senses the spin polarization of the transport current between a sample and a superconducting probe, ${ }^{14,15}$ which has been used to characterize transport spin polarization of various materials. ${ }^{6,16-18}$ Since there is no direct experimental measurement of spin polarization of $\gamma^{\prime}-\mathrm{Fe}_{4} \mathrm{~N}$, we evaluated the transport spin polarization of $\gamma^{\prime}-\mathrm{Fe}_{4} \mathrm{~N}$ using PCAR measurements. To validate the deduced value, it was compared to that of $\alpha$-Fe estimated with an identical experimental setting.

${ }^{a)}$ Electronic mail: suemasu@bk.tsukuba.ac.jp.
$\gamma^{\prime}-\mathrm{Fe}_{4} \mathrm{~N}$ films for spin polarization measurements were prepared by molecular beam epitaxy using $\mathrm{Fe}(\mathrm{CO})_{5}$ as the $\mathrm{Fe}$ source and a nitrogen radical beam produced by electron cyclotron resonance plasma. Approximately $800-\mathrm{nm}$-thick $\gamma^{\prime}-\mathrm{Fe}_{4} \mathrm{~N}$ films were grown. ${ }^{19}$ For comparison, approximately 200 -nm-thick $\alpha$-Fe layers were prepared on $\mathrm{MgO}(001)$ by radio-frequency (rf) magnetron sputtering in an Ar atmosphere at room temperature, followed by coating with $1 \mathrm{~nm}$ Au to prevent surface oxidation of $\alpha$-Fe. The vacuum level was $1.0 \mathrm{~Pa}$ and the rf power was $200 \mathrm{~W}$. The structure of the grown layers was characterized by $\theta-2 \theta$ x-ray diffraction (XRD) using a $\mathrm{Cu} K \alpha$ source. Reflection high-energy electron diffraction (RHEED) patterns were observed along the [100] azimuth of $\mathrm{MgO}$. The spin polarization $(P)$ was measured by the PCAR method using $\gamma^{\prime}-\mathrm{Fe}_{4} \mathrm{~N} / \mathrm{Nb}$ or $\alpha$ - $\mathrm{Fe} / \mathrm{Nb}$ contacts in liquid helium. Several contacts were mechanically made by positioning a sharp needle at various locations on sample surfaces. Conductance-voltage curves were measured across the contacts by the ac four-probe method using a lock-in technique. Interface barriers can be described by introducing a dimensionless interfacial scattering parameter $Z$ into the modified Blonder-Tinkham-Klapwijk (BTK) theory. ${ }^{20,21}$ To estimate the contact diameter of the $\mathrm{Nb}$ tip with the sample and the nature of the electron transport at the point contacts, we used the contact resistance formula from the quasiclassical transport theory which can be applied to both diffusive and ballistic transport as $R=\left(1+Z^{2}\right)$ $\times\left\{\left(4 \rho l_{m} / 3 \pi d^{2}\right)+\gamma\left(l_{m} \rho / 2 d^{2}\right)\right\}$, where $l_{m}=15 \mathrm{~nm}$ is the mean free path of the electron for typical hole conduction in superconductors, $\rho$ is the resistivity $(1.136 \mu \Omega \mathrm{m})$ value measured at $4.2 \mathrm{~K}, d$ is the diameter of the contact, and the prefactor $\gamma$ is usually on the order of unity for mechanical point contacts. ${ }^{22}$ Hence, the typical contact resistance of $20 \Omega$ in this work corresponds to a diameter of approximately $28 \mathrm{~nm}$, which is larger than the average mean free path of electrons in ferromagnets, indicating a diffusive contact. However, physical contact in a point contact is much larger than this value, even though the conductance curve looks almost like that for ballistic transport, which indicates that there might be multiple contacts in the interface contributing simultaneously to the conductance. From our best fitting procedure, the $\Delta$ values were $30 \%$ lower than the bulk 

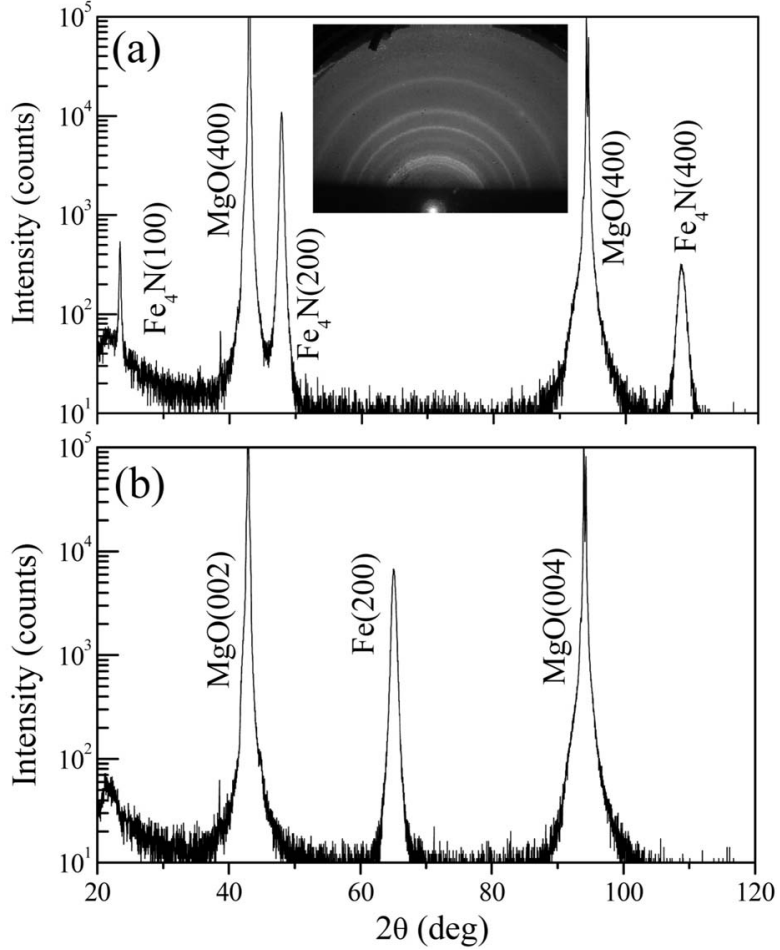

FIG. 1. $\theta-2 \theta$ XRD patterns of (a) $\gamma^{\prime}-\mathrm{Fe}_{4} \mathrm{~N}$ and (b) $\alpha$-Fe films grown on $\mathrm{MgO}(001)$. The inset shows the RHEED pattern of the $\gamma^{\prime}-\mathrm{Fe}_{4} \mathrm{~N}$ film observed along the [100] azimuth of $\mathrm{MgO}$.

superconducting bandgap of $\mathrm{Nb}, 1.5 \mathrm{meV}$. This is thought to result from the multiple contacts that can give rise to suppression of the bandgap as reported by Clowes et al. ${ }^{23}$ for $\mathrm{Nb} / \mathrm{Cu}$; they reported that the gap values were reduced to as low as $0.5 \mathrm{meV}$ as the contact resistance decreased. Woods et $a l .{ }^{24}$ mentioned that the superconductor has a pair-breaking effect due to the interface at the metal/superconductor or the ferromagnet/superconductor, which can be resolved by adopting a temperature higher than the measured value in the fitting procedure. However, the shapes of the conductance curves in both cases are similar. Hence, we have chosen the fitting temperature of $7.8 \mathrm{~K}$, even though it is higher than the measurement temperature. The intrinsic $P$ value was deduced by extrapolating $P$ values for various interfacial scattering parameter $Z$ values to the limit $Z=0$.

Figures 1(a) and 1(b) show typical examples of $\theta-2 \theta$ XRD patterns of $\gamma^{\prime}-\mathrm{Fe}_{4} \mathrm{~N}$ and $\alpha$-Fe films, respectively, grown on $\mathrm{MgO}(001)$ substrates. No peaks other than those from (100)-oriented $\gamma^{\prime}-\mathrm{Fe}_{4} \mathrm{~N}$, that is, the (100), (200), and (400) planes, were observed as shown in Fig. 1(a). The RHEED pattern was not streaky but showed rings, indicating the growth of strongly (100) textured $\gamma^{\prime}-\mathrm{Fe}_{4} \mathrm{~N}$ films with various rotations in the surface normal direction. The formation of (100) textured Fe layers was also confirmed on the $\mathrm{MgO}(001)$ substrate as shown in Fig. 1(b). In our previous paper, ${ }^{19}$ we reported that the epitaxy of $\gamma^{\prime}-\mathrm{Fe}_{4} \mathrm{~N}$ layers on $\mathrm{MgO}(001)$ was enhanced by the deposition of $\alpha$-Fe layers; however, $\alpha$-Fe predeposited layers were not employed in this study.

Figure 2 shows the normalized conductance, $G(V) / G_{\mathrm{N}}$, versus voltage, $V$, curve for the $\gamma^{\prime}-\mathrm{Fe}_{4} \mathrm{~N}$ film measured at 7.8 $\mathrm{K}$ using PCAR. The curve can be fitted by the modified BTK model using three parameters, ${ }^{21}$ namely spin polarization

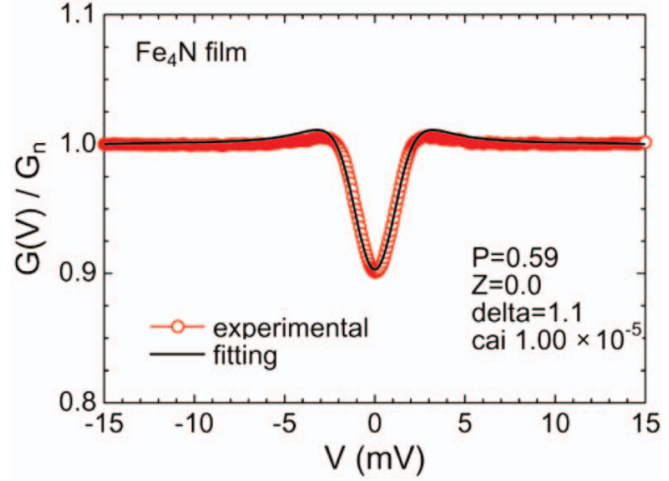

FIG. 2. (Color) Normalized conductance vs voltage curve of $\gamma^{\prime}-\mathrm{Fe}_{4} \mathrm{~N} / \mathrm{Nb}$ contacts measured at $7.8 \mathrm{~K}$. The curve is well fitted at $Z=0.0$. Voltageconductance $\left[V-G(V) / G_{\mathrm{N}}\right]$ curve of the film measured by the PCAR method and the fitted curve by the modified BTK theory using $P, Z$, and $\Delta$ (in $\mathrm{meV}$ ).

$(P)$, the interfacial scattering parameter $(Z)$, and the superconducting bandgap $(\Delta)$, as shown in the figure. According to this model, the $P$ value depends on the $Z$ parameter, and contacts with higher $Z$ result in a lower spin polarization value. Only the $P$ value with a transparent interface $(Z=0)$ corresponds to the intrinsic spin polarization. This scatteringfree state was observed for the $\gamma^{\prime}-\mathrm{Fe}_{4} \mathrm{~N}$ film. As shown in Fig. 2, the curve was fitted well at $Z=0.0$, and thus the $P$ value was directly derived to be 0.59 . This value is as large as those of $\mathrm{Co}_{2} \mathrm{MnSi}$ and $\mathrm{Co}_{2} \mathrm{FeAl},{ }^{25,26}$ which are predicted to be half metals.

To confirm that the nitrogenation of iron enhanced the spin polarization, we measured PCAR spectra from $\alpha$-Fe using the identical setting. Figure 3(a) shows one example of the conductance-voltage curve with $Z=0.11$. In contrast to the curve for $\gamma^{\prime}-\mathrm{Fe}_{4} \mathrm{~N}$, the conductance-voltage curves of the
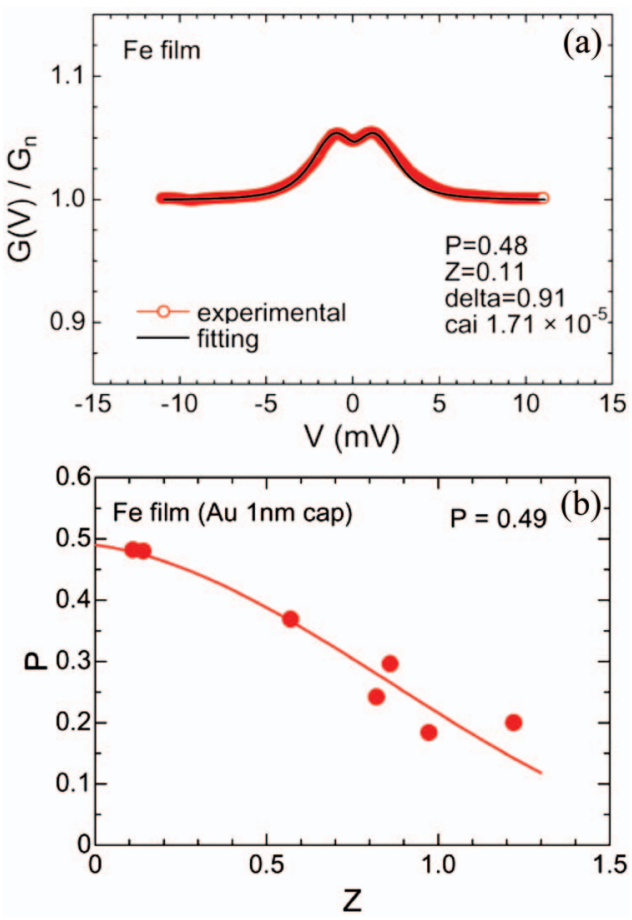

FIG. 3. (Color) (a) Example of normalized conductance vs voltage curve of the $\alpha-\mathrm{Fe} / \mathrm{Nb}$ contacts measured at $7.8 \mathrm{~K}$. The curve is well fitted at $Z$ $=0.11$. (b) Extrapolation to $Z=0$ results in the intrinsic $P$ value. 
$\alpha$-Fe films could not be fitted well using $Z=0.0$. Thus, $P$ values derived for various $Z$ values were measured from several contacts, as shown in Fig. 3(b), and the intrinsic $P$ value was deduced from extrapolating $P$ to $Z=0$. As shown in Fig. 3 , the intrinsic $P$ value was estimated to be approximately 0.49 , which shows reasonable agreement with the previously reported value of 0.44 estimated by superconducting tunneling spectroscopy (STS). ${ }^{27}$ These results have convincingly shown that the spin polarization of $\gamma^{\prime}-\mathrm{Fe}_{4} \mathrm{~N}$ is distinctly higher than that of $\alpha$-Fe. There has been some discussion on the validity of $P$ values derived using modified BTK fitting of PCAR. ${ }^{28}$ However, the $P$ values of $\gamma^{\prime}-\mathrm{Fe}_{4} \mathrm{~N}$ and $\alpha$-Fe films were obtained by the same PCAR technique with an identical setting, and the conductance versus voltage curves were well fitted with the modified BTK mode in this work. In addition, the obtained $P$ value for $\alpha$-Fe is close to that reported previously using STS. Thus, it can at least be stated that the spin polarization of $\gamma^{\prime}-\mathrm{Fe}_{4} \mathrm{~N}$ is significantly larger than that of $\alpha$-Fe. According to Ref. 9, the large spin polarization in $\gamma^{\prime}-\mathrm{Fe}_{4} \mathrm{~N}$ can be attributed to the enhanced transport of $3 d$ bands by introducing an $\mathrm{N}$ atom at the body center position of $\gamma$-Fe (fcc structure). However, we do not have sufficient data to discuss the underlying mechanism. Therefore, further experimental studies on the DOSs in $\gamma^{\prime}-\mathrm{Fe}_{4} \mathrm{~N}$ together with TMR ratios in MTJs and MR ratios in CPP GMR would be useful for understanding the mechanism of large spin polarization of $\gamma^{\prime}-\mathrm{Fe}_{4} \mathrm{~N}$.

In summary, we have estimated the spin polarization of $\gamma^{\prime}-\mathrm{Fe}_{4} \mathrm{~N}$ and $\alpha$-Fe films by the PCAR method to investigate the possibility of enhancement of spin polarization by nitrogenation of Fe. The conductance versus voltage curves were well fitted with the modified BTK model to deduce spin polarization values of $\gamma^{\prime}-\mathrm{Fe}_{4} \mathrm{~N}$ and $\alpha$-Fe films to be 0.59 and 0.49 , respectively, at $7.8 \mathrm{~K}$. The spin polarization value of 0.59 for $\gamma^{\prime}-\mathrm{Fe}_{4} \mathrm{~N}$ is distinctly larger than that of $\mathrm{Fe}$ and is comparable to that of $\mathrm{Co}_{75} \mathrm{Fe}_{25}{ }^{22}$

This work was supported in part by the World Premier International Research Center (WPI) Initiative on Materials Nanoarchitronics, MEXT, Grant-in-Aid for Scientific Research on Priority Area on "Creation and Control of Spin Current” Grant No. 19048029 from MEXT, PREST-JST, and the NanoProcessing Partnership Platform (NPPP) at AIST, Tsukuba.
${ }^{1}$ R. A. de Groot, F. M. Muller, P. G. van Engen, and K. H. J. Bushow, Phys. Rev. Lett. 50, 2024 (1983).

${ }^{2}$ I. Galanakis, J. Phys.: Condens. Matter 14, 6329 (2002).

${ }^{3}$ S. Kammerer, A. Thomas, A. Hutten, and G. Reiss, Appl. Phys. Lett. 85, 79 (2004).

${ }^{4}$ X. Y. Dong, C. Adelmann, J. Q. Xie, C. J. Palmstrom, X. Lou, J. Strand, P. A. Crowell, J.-P. Barnes, and K. Petford-Long, Appl. Phys. Lett. 86, 102107 (2005).

${ }^{5}$ Y. Sakuraba, M. Hattori, M. Oogane, Y. Ando, H. Kato, A. Sakuma, T. Miyazaki, and H. Kubota, Appl. Phys. Lett. 88, 192508 (2006).

${ }^{6}$ Z. Gercsi, A. Rajanikanth, Y. K. Takahashi, and K. Hono, Appl. Phys. Lett. 89, 082512 (2006).

${ }^{7}$ S. Karthik, A. Rajanikanth, Y. K. Takahashi, T. Ohkubo, and K. Hono, Acta Mater. 55, 3867 (2007)

${ }^{8}$ Y. Miura, H. Uchida, Y. Oba, K. Abe, and M. Shirai, Phys. Rev. B 78, 064416 (2008).

${ }^{9}$ S. Kokado, N. Fujima, K. Harigawa, H. Shimizu, and A. Sakuma, Phys. Rev. B 73, 172410 (2006).

${ }^{10}$ K. Sunaga, M. Tsunoda, K. Komagaki, Y. Uehara, and M. Takahashi, J. Appl. Phys. 102, 013917 (2007).

${ }^{11}$ Y. Komasaki, M. Tsunoda, S. Isogami, and M. Takahashi, J. Appl. Phys. 105, 07C928 (2009).

${ }^{12}$ M. Julliere, Phys. Lett. 54A, 225 (1975).

${ }^{13}$ J. M. MacLaren, X.-G. Zhang, and W. H. Butler, Phys. Rev. B 56, 11827 (1997).

${ }^{14}$ S. K. Upadhyay, A. Palanisami, R. N. Louie, and R. A. Buhrman, Phys. Rev. Lett. 81, 3247 (1998).

${ }^{15}$ R. J. Soulen, Jr., J. M. Byers, M. S. Osofsky, B. Nadgorny, T. Ambrose, S. F. Cheng, P. R. Broussard, C. T. Tanaka, J. Nowak, J. S. Moodera, A. Barry, and J. M. D. Coey, Science 282, 85 (1998).

${ }^{16}$ Y. Ji, G. J. Strijkers, F. Y. Yang, C. L. Chien, J. M. Byers, A. Anguelouch, G. Xiao, and A. Gupta, Phys. Rev. Lett. 86, 5585 (2001).

${ }^{17}$ J. M. Valentine, and C. L. Chien, J. Appl. Phys. 99, 08 P902 (2006).

${ }^{18}$ S. V. Karthik, T. M. Nakatani, A. Rajanikanth, Y. K. Takahashi, and K. Hono, J. Appl. Phys. 105, 07C916 (2009).

${ }^{19}$ A. Narahara, K. Ito, and T. Suemasu, J. Cryst. Growth 311, 1616 (2009).

${ }^{20}$ G. E. Blonder, M. Tinkham, and T. M. Klapwijk, Phys. Rev. B 25, 4515 (1982)

${ }^{21}$ G. J. Strijkers, Y. Ji, F. Y. Yang, C. L. Chien, and J. M. Byers, Phys. Rev. B 63, 104510 (2001).

${ }^{22}$ B. Nikolic and P. B. Allen, Phys. Rev. B 60, 3963 (1999).

${ }^{23}$ S. K. Clowes, Y. Miyoshi, O. Johannson, B. J. Hickey, C. H. Marrows, M. Blamire, M. R. Branford, Y. V. Bugoslavsky, and L. F. Cohen, J. Magn. Magn. Mater. 272-276, E1471 (2004).

${ }^{24}$ G. T. Woods, R. J. Soulen, Jr., I. Mazin, B. Nadgorny, M. S. Osofsky, J. Sanders, H. Srikanth, W. F. Egelhoff, and R. Datla, Phys. Rev. B 70, 054416 (2004).

${ }^{25}$ L. Ritchie, G. Xiao, Y. Ji, Y. Chen, C. L. Chien, M. Zhang, J. Chen, Z. Liu, and G. Wu, Phys. Rev. B 68, 104430 (2003).

${ }^{26}$ T. M. Nakatani, A. Rajanikanth, Z. Gercsi, Y. K. Takahashi, K. Inomata, and K. Hono, J. Appl. Phys. 102, 033916 (2007).

${ }^{27}$ P. M. Tedrow and R. Meservey, Phys. Rev. B 7, 318 (1973).

${ }^{28}$ Y. Bugoslavsky, Y. Miyoshi, S. K. Clowes, W. R. Branford, M. Lake, I. Brown, A. D. Caplin, and L. F. Cohen, Phys. Rev. B 71, 104523 (2005). 